\title{
LETTER
}

\section{Clinical phenotypes of critically ill COVID-19 patients}

\author{
Elie Azoulay* ${ }^{*}$, Lara Zafrani, Adrien Mirouse, Etienne Lengliné, Michael Darmon and Sylvie Chevret
}

(ㄷ) 2020 Springer-Verlag GmbH Germany, part of Springer Nature

\section{Dear Editor,}

Patients with severe acute respiratory syndrome due to coronavirus 2 (SARS-CoV-2) require intensive care unit (ICU) admission for hypoxemic respiratory failure [1]. The COVID-19 pandemic has led to shortages of essential material and human resources that mandate practices such as triage and rationing [2]. Little information on this new disease is available to help clinicians identify, at the time they make the decision of ICU admission, the patients who have severe hypoxemia but a relatively low risk of intubation or death if admitted to the ICU.

We report the clinical and laboratory features in an observational cohort of 85 consecutive COVID-19 patients admitted to an ICU in Paris, France, between March 7 and April 6, 2020. Reverse-transcriptase polymerase chain reaction (RT-PCR) assays of nasopharyngeal samples were positive for SARS-CoV-2 in all 85 patients. The median age was 61 (53-67) years, 62 (74\%) patients were men, and the median time from flu-like symptom onset to ICU admission was 8 (6-11) days. Body mass index was 28 (25-31); 26 (31\%) patients had diabetes, 41 (48\%) hypertension, and 19 (22\%) immune deficiencies. At ICU admission, oxygen flow was $12 \mathrm{~L} /$ min (6-15). Overall, 43 (51\%) patients were intubated and $18(21 \%)$ died in the ICU.

Hierarchical clustering in a principal component approach identified three clinical and biological phenotypes at ICU admission (Fig. 1), as follows. Cluster 1 $(N=37,43.5 \%$, low mortality) was characterized by a predominance of females; $9 \mathrm{~L} / \mathrm{min}$ oxygen at presentation; relatively low ferritin, D-dimers, and CRP levels but intermediate IL6 levels; 8\% ICU mortality. Cluster $2(N=17,20 \%$, intermediate mortality) had younger patients; $88 \%$ males; no fever; criteria for acute kidney injury in $87 \%$ of cases; $6 \mathrm{~L} / \mathrm{min}$ oxygen at presentation; low IL6 concentrations but intermediate ferritin, D-dimers, and CRP; 18\% ICU mortality. Cluster $3(N=31,36.5 \%$, high mortality) was remarkable for the marked majority of men; older age than in the other two clusters; severe hypoxemia with $15 \mathrm{~L} /$ min oxygen at presentation; fever; intense inflammatory syndrome with high CRP, D-dimer, ferritin, and IL6 concentrations; 39\% ICU mortality. Intubation rates were $41 \%, 33 \%$, and $71 \%$ in clusters 1,2 , and 3 , respectively.

By multivariable analysis (Cox proportional hazards model), factors associated with the need for intubation and mechanical ventilation were age $>65$ years (HR 1-3.51) and oxygen flow at presentation (HR 1.02/l (1-1.04)). Determinants of ICU mortality were age $>70$ years (HR 1.41-17.92) and the number of comorbid conditions (HR 1.09-2.81).

This clustering approach correlates initial clinical features and outcomes in 85 critically ill hypoxemic COVID19 patients. Awareness of these clusters may assist clinicians in making ICU admission decisions when beds are scarce. Moreover, the marked variations in inflammation markers across clusters suggest possible differences in the response to specific COVID-19 treatment $[3,4]$. 
Patient's characteristics at the time of ICU admission

a

\begin{tabular}{lllll}
\hline \hline Medial (IQR) & Cluster 1 & Cluster 2 & Cluster 3 & $\mathbf{P}$ \\
or N (\%) & $\mathbf{N}=37$ & $\mathrm{~N}=17$ & $\mathrm{~N}=31$ & value \\
\hline Age (y) & $61(42-64)$ & $57(53-62)$ & $64(56-70)$ & 0.04 \\
Gender (female) & $16(43 \%)$ & $2(12 \%)$ & $4(13 \%)$ & 0.008 \\
Body Mass Index & $29.5(26-32)$ & $26(25-31)$ & $26(24-29)$ & 0.06 \\
Number of comorbidities & $2(1-3)$ & $3(2-5)$ & $2(1-3)$ & 0.12 \\
Time since flu symptoms & $8(5-10)$ & $10(6-15)$ & $7(5-10)$ & 0.23 \\
Oxygen flow at presentation & $9(9-15)$ & $6(3-15)$ & $15(9-15)$ & 0.03 \\
Respiratory rate & $27(22-31)$ & $28(25-34)$ & $30(24-34)$ & 0.22 \\
Temperature at presentation & $38.3(37.8-38.9)$ & $37(36.9-37.4)$ & $38(37-38.8)$ & 0.002 \\
Acute kidney injury & $1(3.4)$ & $14(87)$ & $14(56)$ & $<0.0001$ \\
C Reactive Protein (/mI) & $119(75-198)$ & $148(103-268)$ & $277(233-321)$ & $<0.0001$ \\
Fibrinogen & $6.00(5.40-7.00)$ & $6.7(5.97-7.17)$ & $7.45(6.72-7.88)$ & 0.0006 \\
D dimers & $820(670-1360)$ & $1440(930-7390)$ & $2300(1510-3630)$ & 0.0004 \\
Interleukin 6 & $69(47-94)$ & $32(21-40)$ & $155(102-294)$ & $<0.0001$ \\
Ferritin & $786(498-1438)$ & $1822(1296-2371)$ & $1693(941-3572)$ & 0.002 \\
Need for intubation & $15(41)$ & $6(35)$ & $22(71)$ & 0.03 \\
ICU mortality & $3(8)$ & $3(18)$ & $12(39)$ & 0.007 \\
\hline \hline & & & & \\
\hline
\end{tabular}

b

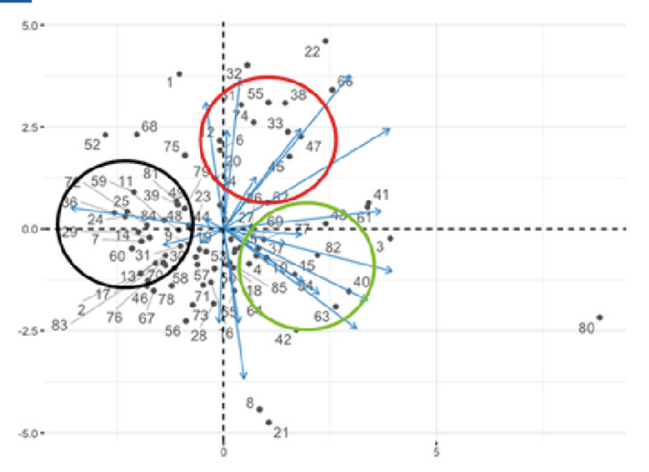

C

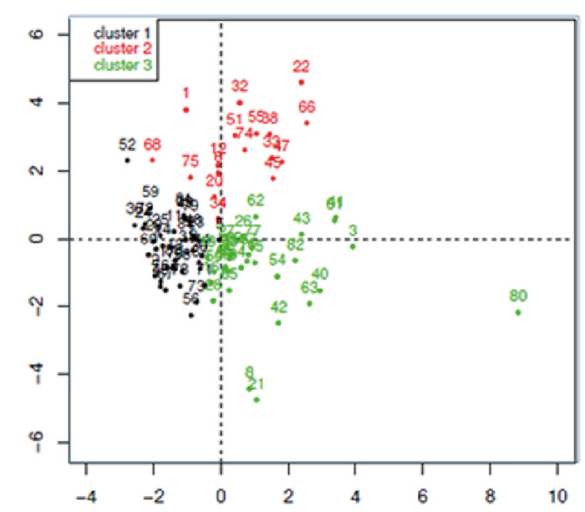

Fig. 1 Patients' characteristics and results of the hierarchical clustering disclose three distinct clusters. a Patient's characteristics at the time of ICU admission. b Principal Components Analysis of parameters available at ICU admission. c Factor map showing the distribution of each patient in each cluster

\section{Compliance with ethical standards}

\section{Conflicts of interest}

None of the authors has any conflicts of interest to declare.

\section{Publisher's Note}

Springer Nature remains neutral with regard to jurisdictional claims in published maps and institutional affiliations.

Accepted: 14 May 2020

Published online: 28 May 2020

\section{References}

1. Murthy S, Gomersall CD, Fowler RA (2020) Care for critically ill patients with COVID-19. JAMA. https://doi.org/10.1001/jama.2020.3633

2. Truog RD, Mitchell C, Daley GQ (2020) The toughest triage_allocating ventilators in a pandemic. N Engl J Med 382(21):1973-1975. https://doi. org/10.1056/NEJMp2005689

3. Sanders JM, Monogue ML, Jodlowski TZ, Cutrell JB (2020) Pharmacologic treatments for coronavirus disease 2019 (COVID-19): a review. JAMA. https://doi.org/10.1001/jama.2020.6019

4. Alhazzani W, Møller MH, Arabi YM et al (2020) Surviving Sepsis Campaign: guidelines on the management of critically ill adults with Coronavirus Disease 2019 (COVID-19). Intensive Care Med 46:854-887 